\title{
Anatomy of health effects of Mediterranean diet: Greek EPIC prospective cohort study
}

\author{
Antonia Trichopoulou, professor, ${ }^{1}$ Christina Bamia, lecturer, ${ }^{1}$ Dimitrios Trichopoulos, professor $^{2}$
}

\begin{abstract}
${ }^{1}$ Department of Hygiene,
Epidemiology and Medical

Statistics, University of Athens,

Medical School, 11527 Athens,

Greece

${ }^{2}$ Department of Epidemiology, Harvard School of Public Health, 677 Huntington Avenue, Boston, MA 02115, USA

Correspondence to: D

Trichopoulos

dtrichop@hsph.harvard.edu
\end{abstract}

Cite this as: $B M J$ 2009;338:b2337 doi:10.1136/bmj.b2337

\begin{abstract}
Objective To investigate the relative importance of the individual components of the Mediterranean diet in generating the inverse association of increased adherence to this diet and overall mortality.

Design Prospective cohort study.

Setting Greek segment of the European Prospective Investigation into Cancer and nutrition (EPIC).

Participants 23349 men and women, not previously diagnosed with cancer, coronary heart disease, or diabetes, with documented survival status until June 2008 and complete information on nutritional variables and important covariates at enrolment.
\end{abstract}

Main outcome measure All cause mortality.

Results After a mean follow-up of 8.5 years, 652 deaths from any cause had occurred among 12694 participants with Mediterranean diet scores 0-4 and 423 among 10655 participants with scores of 5 or more. Controlling for potential confounders, higher adherence to a Mediterranean diet was associated with a statistically significant reduction in total mortality (adjusted mortality ratio per two unit increase in score $0.864,95 \%$ confidence interval 0.802 to 0.932 ). The contributions of the individual components of the Mediterranean diet to this association were moderate ethanol consumption $23.5 \%$, low consumption of meat and meat products $16.6 \%$, high vegetable consumption $16.2 \%$, high fruit and nut consumption $11.2 \%$, high monounsaturated to saturated lipid ratio $10.6 \%$, and high legume consumption $9.7 \%$. The contributions of high cereal consumption and low dairy consumption were minimal, whereas high fish and seafood consumption was associated with a non-significant increase in mortality ratio.

Conclusion The dominant components of the Mediterranean diet score as a predictor of lower mortality are moderate consumption of ethanol, low consumption of meat and meat products, and high consumption of vegetables, fruits and nuts, olive oil, and legumes. Minimal contributions were found for cereals and dairy products, possibly because they are heterogeneous categories of foods with differential health effects, and for fish and seafood, the intake of which is low in this population.

\section{INTRODUCTION}

The Mediterranean diet was introduced to the scientific community as a health protecting diet by the classic studies of Ancel Keys and colleagues. ${ }^{1}$ In 1995 a simple score to assess adherence to the Mediterranean diet was introduced, ${ }^{2}$ and this score, or variants of it, has been used to evaluate the relation of the Mediterranean diet to overall mortality, as well as to specific health outcomes, including Alzheimer's disease, diabetes mellitus, and cancer overall..$^{3-5}$ Most of these studies have focused on overall survival, as this is a natural priority. A recent meta-analysis summarised the results of nine cohort studies that evaluated the relation between adherence to the Mediterranean diet and overall mortality. ${ }^{6}$ An inverse association was noted in all these studies, with a pooled mortality ratio (relative risk) of 0.91 (95\% confidence interval 0.89 to 0.94 ) for each two point increment in a scale ranging from 0 to 9 . The reported associations were somewhat stronger in studies done in Mediterranean countries and somewhat weaker in studies done in western European countries and Sweden, ${ }^{2-10}$ although no statistically significant heterogeneity existed. ${ }^{6}$ However, no attempt has been made to investigate the relative importance of the individual components of the Mediterranean diet (and of the Mediterranean diet score) in the generation of the inverse association of increased adherence to this diet and overall mortality.

We have evaluated the contribution of the nine widely accepted components of the Mediterranean diet (high intake of vegetables, fruits and nuts, legumes, fish and seafood, and cereals; low intake of meat and meat products and dairy products; high ratio of monounsaturated to saturated lipids; and moderate intake of ethanol) in the inverse association of this diet with all cause mortality in a population based cohort in Greece.

\section{METHODS}

European Prospective Investigation into Cancer and nutrition

The study population consisted of the participants in the Greek segment of the European Prospective Investigation into Cancer and nutrition (EPIC). EPIC is run in 23 research centres across 10 European countries, with the purpose of investigating the role of biological, 
Table 1| Distribution of study participants without cancer, coronary heart disease, or diabetes mellitus at enrolment, by sex and baseline sociodemographic, somatometric, lifestyle, and medical characteristics. Values are numbers (percentages)

\begin{tabular}{|c|c|c|c|}
\hline Characteristic & Men $(n=9504)$ & Women $(n=13845)$ & Total $(n=23349)$ \\
\hline \multicolumn{4}{|l|}{ Age (years): } \\
\hline$<45$ & 3479 (37) & $4521(33)$ & 8000 (34) \\
\hline $45-54$ & $2361(25)$ & $3402(25)$ & $5763(25)$ \\
\hline $55-64$ & $1923(20)$ & $3338(24)$ & $5261(23)$ \\
\hline$\geq 65$ & 1741 (18) & 2584 (19) & 4325 (19) \\
\hline \multicolumn{4}{|l|}{ Education: } \\
\hline $\begin{array}{l}\text { None/elementary school } \\
\text { degree }\end{array}$ & $4471(47)$ & $8295(60)$ & $12766(55)$ \\
\hline $\begin{array}{l}\text { Secondary/technical } \\
\text { school degree }\end{array}$ & 2994 (32) & 3247 (23) & $6241(27)$ \\
\hline University degree/higher & $2039(21)$ & $2303(17)$ & 4342 (19) \\
\hline \multicolumn{4}{|l|}{$\begin{array}{l}\text { Smoking of cigarettes (at } \\
\text { enrolment): }\end{array}$} \\
\hline Current & 3904 (41) & $2543(18)$ & 6447 (28) \\
\hline Former & 2964 (31) & $1101(8)$ & 4065 (17) \\
\hline Never & $2636(28)$ & $10201(74)$ & $12837(55)$ \\
\hline \multicolumn{4}{|l|}{ Body mass index $\left(\mathrm{kg} / \mathrm{m}^{2}\right)$ : } \\
\hline$\leq 25$ & $1897(20)$ & $3545(26)$ & $5442(23)$ \\
\hline$>25-\ll 30$ & $5006(53)$ & $5152(37)$ & $10158(44)$ \\
\hline$\geq 30$ & $2601(27)$ & $5148(37)$ & 7749 (33) \\
\hline \multicolumn{4}{|l|}{ Waist:hip ratio: } \\
\hline$\leq 0.90$ & $2082(22)$ & $11948(86)$ & $14030(60)$ \\
\hline$>0.90<<0.95$ & $2694(28)$ & $1155(8)$ & 3849 (16) \\
\hline$\geq 0.95$ & $4728(50)$ & $742(5)$ & $5470(23)$ \\
\hline \multicolumn{4}{|l|}{$\begin{array}{l}\text { Physical activity (MET- } \\
\text { hours/day): }\end{array}$} \\
\hline$\ll 34.996$ & $5109(54)$ & $6567(47)$ & $11676(50)$ \\
\hline$\geq 34.996$ & $4395(46)$ & $7278(53)$ & $11673(50)$ \\
\hline \multicolumn{4}{|l|}{ Ethanol intake*: } \\
\hline Low & $4484(47)$ & $10900(79)$ & $15384(66)$ \\
\hline Moderate & $4200(44)$ & $2731(20)$ & $6931(30)$ \\
\hline High & $820(9)$ & $214(2)$ & $1034(4)$ \\
\hline
\end{tabular}

*Low: $<10 \mathrm{~g} /$ day for men, $<5 \mathrm{~g} /$ day for women; moderate: men $\geq 10 \mathrm{~g} /$ day and $\leq 50 \mathrm{~g} /$ day, women $\geq 5 \mathrm{~g} /$ day and $\leq 25 \mathrm{~g} /$ day; high: $>50 \mathrm{~g} /$ day for men, $>25 \mathrm{~g} /$ day for women.

dietary, lifestyle, and environmental factors in causing cancer and other chronic diseases. ${ }^{11} 12$

In Greece, 28572 apparently healthy men and women aged 20-86 years were recruited from the general population throughout the country between 1994 and 1997. Trained interviewers administered dietary and lifestyle questionnaires to participants at enrolment, and trained health professionals made somatometric measurements.

\section{Data on diet}

Dietary intakes during the year preceding enrolment were assessed with the use of a validated food frequency questionnaire that includes approximately 150 foods and beverages commonly consumed in Greece. ${ }^{13}$ Standard portion sizes were used for the estimation of consumed quantities, and nutrient and ethanol intakes were calculated by using a food composition database modified to accommodate the particularities of the Greek diet. ${ }^{14}$ For each participant, grams per day of intake of various food groups and nutrients, as well as total energy intake, were calculated.

For this analysis, we focused on nine nutritional variables: vegetables, legumes, fruits and nuts, dairy products, cereals, meat and meat products, fish and seafood, monounsaturated to saturated lipid ratio, and ethanol.

\section{Mediterranean diet score}

We assessed the conformity to the traditional Mediterranean diet with a 10 unit scale. $^{7}$ The scale relies on nine dietary components that capture the essence of the traditional Mediterranean diet. Vegetables, legumes, fruits and nuts, fish and seafood, and cereals are presumed to be beneficial for health, whereas meat and meat products and dairy products are presumed not to be beneficial. A higher ratio of monounsaturates to saturates is considered to be beneficial and reflects the high olive oil consumption that characterises the traditional Mediterranean diet. Ethanol is used as a measure of the consumption of alcoholic beverages, which in the Mediterranean countries are mostly consumed during meals and mainly in the form of wine.

We assigned values of 0 or 1 to each of the above indicated components (except for ethanol intake), using the sex specific medians in the studied population as cut-offs. We assigned a value of 0 to people whose consumption was below the median values of components with a presumably beneficial effect and a value of 1 to people with consumption equal to or above the median. In contrast, we assigned a value of 1 to people with below the median consumption of components without a beneficial effect and a value of 0 to those whose consumption of these components was above the corresponding median. For ethanol, we gave value of 1 to men who consumed quantities from $10 \mathrm{~g}$ (or one unit) a day to less than $50 \mathrm{~g}$ (or six units) a day and a value of 0 otherwise; the corresponding cut-offs for women were $5 \mathrm{~g}$ (or half a unit) a day and $25 \mathrm{~g}$ (or three units) a day (in the United Kingdom, a unit of alcohol is defined as $10 \mathrm{ml}$, or approximately $8 \mathrm{~g}$, of ethanol). Thus, the total Mediterranean diet score can take values from 0 (minimal conformity to the traditional Mediterranean diet) to 9 (maximal conformity to the traditional Mediterranean diet).

Lifestyle, anthropometric, dietary, and medical history data A section of the lifestyle questionnaire recorded the frequency and duration of participation in occupational and leisure time physical activities. We assigned a multiple of resting metabolic rate (metabolic equivalent of task or MET value) to each of these activities. We then multiplied time spent on each activity by the MET value of the activity and summed the resulting MET-hour products to produce an index of daily physical activity, expressing the amount of energy per kilogram of body weight expended during an average day. ${ }^{15}$

Information on smoking status was collected through a smoking history questionnaire. We categorised participants as never, current, and former 
smokers as of the date of enrolment. For current smokers, we recorded the average number of cigarettes smoked each day. ${ }^{16}$ Standardised procedures were used to make anthropometric measurements. ${ }^{17} \mathrm{We}$ derived body mass index as the ratio of weight in kilograms divided by the square of the height in metres and calculated the waist to hip ratio. Participants were also asked whether they had ever had a medically documented diagnosis of certain diseases - specifically, cancer, diabetes mellitus, myocardial infarction, and angina pectoris.

\section{Study participants and follow-up}

Greek EPIC participants were actively followed up by teams led by trained physicians until June 2008. Of the initial 28572 participants, $1073(4 \%)$ did not respond or were not traced during follow-up and were excluded from further analysis. Of the remaining 27499 participants, 3554 reported at enrolment that they had had a previous diagnosis of coronary heart disease $(\mathrm{n}=1371)$, cancer (611), or diabetes mellitus (1921). For an additional 596 participants, information was missing for one or more of the sociodemographic, dietary, medical, somatometric, or lifestyle variables used in this investigation. Thus, the final sample used for the analyses reported in this paper consisted of 23349 participants who had not previously been diagnosed as having cancer, coronary heart disease, or diabetes mellitus; for whom vital status at follow-up had been ascertained; and for whom all the required information was available.

The mean length of follow-up for the 23349 study participants was 8.5 years. The range was 10 days (a participant who died soon after enrolment) to 14 . 2 years.

\section{Statistical analysis}

We used Stata statistical software for all analyses. We set statistical significance at two sided $\mathrm{P}<0.05$. We present descriptive sociodemographic data by simple tabulations and dietary variables as medians and interquartile ranges.

\begin{tabular}{lcc}
\hline $\begin{array}{l}\text { Table } \mathbf{2} \text { |Daily intakes (g/day) of indicated dietary variables by sex. Values are median } \\
\text { (interquartile range) }\end{array}$ & $\begin{array}{c}\text { Men } \\
\text { Dietary variable }\end{array}$ & Women \\
\begin{tabular}{l} 
Vegetables \\
\hline Legumes
\end{tabular} & $548.60(434.67-683.86)$ & $499.33(388.98-634.26)$ \\
\hline Fruits and nuts & $362.52(257.17-45-13.32)$ & $6.66(3.62-10.52)$ \\
\hline Cereals & $178.32(135.24-232.98)$ & $356.77(257.15-471.46)$ \\
\hline Fish and seafood & $23.67(13.92-33.62)$ & $139.59(108.06-176.22)$ \\
\hline Dairy products & $196.11(116.90-301.35)$ & $18.89(12.51-27.73)$ \\
\hline Meat and meat products & $121.11(86.83-163.69)$ & $89.88(64.02-120.09)$ \\
\hline Monounsaturated lipids & $56.04(44.67-69.45)$ & $46.62(36.92-57.86)$ \\
\hline Saturated lipids & $33.08(25.19-42.06)$ & $27.11(20.62-34.83)$ \\
\hline $\begin{array}{l}\text { Monounsaturated/saturated lipids } \\
\text { (ratio) }\end{array}$ & $1.72(1.46-2.02)$ & $1.74(1.45-2.07)$ \\
\hline Ethanol & $11.07(3.43-25.41)$ & $1.17(0.04-4.02)$ \\
\hline
\end{tabular}

We used proportional hazards (Cox) regression models to analyse survival data. In these models, the time variable was the interval between the date of enrolment and the date of last follow-up or date of death, whichever occurred first. Participants who were alive as of the date of last follow-up or were lost to follow-up were considered censored as of the date of last contact.

With the Cox regression models, we estimated the association ("effect") of a two unit increase in the Mediterranean diet score with all cause mortality. We also evaluated the relative importance of each of the components of the scale as follows. Firstly, we included all nine components, considered as dichotomous (using the sex specific medians as cut-offs), simultaneously in a Cox regression. Subsequently, we evaluated the influence of each of the dietary components on the mortality ratio associated with the Mediterranean diet score by subtracting alternately one component at a time from the original score (thus reducing the 10 point score to nine point scores) and estimating the nine mortality ratios associated with a two unit increment in the score minus vegetables, score minus pulses, score minus fruits and nuts, score minus cereals, score minus fish and seafood, score minus lipid ratio, score minus meat and meat products, score minus dairy products, and score minus ethanol. To preserve comparability, we multiplied the logarithm of the estimated nine mortality ratios by $9 / 10$ before exponentiating them.

In another analysis, we successively removed from the Mediterranean diet score each of the components in descending order of importance (as assessed from the model in which all nine components were simultaneously included) to evaluate the impact on the mortality ratio of the sequential removal of the component factors. Because successive removal of components reduces the range of the score, and to preserve comparability, we multiplied each of the successively derived $\log$ coefficients by $9 / 10,8 / 10,7 / 10$, and so on before exponentiating them.

Lastly, we evaluated the association with mortality of the joint action of each two by two combination of the individual components of the Mediterranean diet score and examined whether the joint action of any combination of two components was at least additive. As the Mediterranean score includes nine dietary components, we assessed $36(9 \times(9-1) / 2)$ two by two combinations. In these combinations, we dichotomised each dietary component as indicated in the construction of the Mediterranean diet score.

In all analyses, we controlled for sex, age at enrolment $(<45,45-54,55-64, \geq 65$ years; categorically), education (none/elementary school degree, secondary school or technical school degree, university degree or higher; categorically), smoking status (never, former, and, current at enrolment with cigarettes per day, 1-10, 11-20, 21-30, 31-40, $\geq 41$; ordered), MET-hours (fifths; ordered), total energy intake (fifths; ordered), waist to hip ratio (sex specific fifths; ordered), and body mass index (sex specific fifths; ordered). 
Table $3 \mid$ Mutually adjusted mortality ratios associated with intake of components of Mediterranean diet

\begin{tabular}{|c|c|c|}
\hline Dietary variable & Mortality ratio* $(95 \% \mathrm{Cl})$ & $P$ value \\
\hline \multicolumn{3}{|l|}{ Vegetables: } \\
\hline 〈Median & Reference & \\
\hline$\geq$ Median & 0.901 (0.775 to 1.048$)$ & 0.177 \\
\hline \multicolumn{3}{|l|}{ Legumes: } \\
\hline$<$ Median & Reference & \\
\hline$\geq$ Median & $0.942(0.825$ to 1.076$)$ & 0.379 \\
\hline \multicolumn{3}{|l|}{ Fruits and nuts: } \\
\hline «Median & Reference & \\
\hline$\geq$ Median & 0.931 (0.816 to 1.061$)$ & 0.284 \\
\hline \multicolumn{3}{|l|}{ Cereals: } \\
\hline <Median & Reference & \\
\hline$\geq$ Median & $0.989(0.862$ to 1.133$)$ & 0.869 \\
\hline \multicolumn{3}{|l|}{ Fish and seafood } \\
\hline <Median & Reference & \\
\hline$\geq$ Median & $1.078(0.950$ to 1.224$)$ & 0.243 \\
\hline \multicolumn{3}{|c|}{$\begin{array}{l}\text { Monounsaturated/saturated lipids } \\
\text { (ratio): }\end{array}$} \\
\hline «Median & Reference & \\
\hline$\geq$ Median & 0.908 (0.792 to 1.042$)$ & 0.171 \\
\hline \multicolumn{3}{|l|}{ Dairy products: } \\
\hline$<$ Median & Reference & \\
\hline$\geq$ Median & 1.069 (0.931 to 1.227$)$ & 0.345 \\
\hline \multicolumn{3}{|c|}{ Meat and meat products: } \\
\hline <Median & Reference & \\
\hline$\geq$ Median & $1.148(0.992$ to 1.329$)$ & 0.063 \\
\hline \multicolumn{3}{|l|}{ Ethanol intake†: } \\
\hline Moderate & Reference & \\
\hline Low intake & $1.193(1.032$ to 1.380$)$ & 0.017 \\
\hline High intake & 1.470 (1.132 to 1.907$)$ & 0.004 \\
\hline
\end{tabular}

*Adjusted for sex, age ( $<45,45-54,55-64, \geq 65$, categorically), education (none/elementary school degree, secondary or technical school degree, university degree or higher, categorically), smoking status (never, former, and current at enrolment with cigarettes per day 1-10, 11-20, 21-30, 31-40, $\geq 41$ ordered), waist:hip ratio (sex specific fifths, ordered), body mass index (sex specific fifths, ordered), MET score (fifths, ordered), and total energy intake (fifths, ordered).

†Low: $<10 \mathrm{~g} /$ day for men, $<5 \mathrm{~g} /$ day for women; moderate: men $\geq 10 \mathrm{~g} /$ day and $\leq 50 \mathrm{~g} /$ day, women $\geq 5 \mathrm{~g} /$ day and $\leq 25 \mathrm{~g} /$ day; high: $>50 \mathrm{~g} /$ day for men, $>25 \mathrm{~g} /$ day for women.

Whenever we excluded one or more factors from the Mediterranean diet score, we still adjusted for these factors in the statistical analyses to control for possible confounding. Moreover, when we evaluated the two by two combinations, we controlled for the rest of the components of the original score. We checked the proportionality assumption with the log-log plots.

\section{RESULTS}

The 23349 study participants were followed up for 199726 person years, during which period 1075 deaths occurred. Table 1 shows the distribution of study participants without a medically documented history of major chronic diseases at enrolment. By design, the Greek EPIC cohort contains more women than men. The sampled age groups contain relatively few people with university level education. As is well known for the Greek population in general, a high proportion of people were overweight or obese and a high proportion of men were ever smokers. Almost half of men and a substantial fraction of women were consumers of a moderate amount of alcoholic beverages; few men and very few women were high consumers.

Table 2 shows medians and interquartile ranges of the daily intakes (by sex) of food groups that are characteristics of the traditional Mediterranean diet because they are consumed either in relatively high or in relatively low amounts. The high consumption of vegetables, legumes, fruits, and monounsaturated lipids (mostly olive oil) is evident.

Table 3 shows associations, derived from Cox regression, of the nine components of the Mediterranean diet with mortality, assessed through mutually adjusted ratios contrasting high with low consumption (except for ethanol) and controlled for potential confounders as indicated in the footnote. All components of the Mediterranean diet score have been simultaneously introduced in this model, allowing assessment of the relative impact of each component. Compared with moderate intake of ethanol, both low and high intakes were associated with excess mortality to a statistically significant degree. Among the presumed beneficial components of the Mediterranean diet score, high consumption of all but fish and seafood was inversely associated with mortality, although none of these associations was statistically significant. For fish and seafood, the mortality ratio for consumption above or equal to the median compared with consumption below the median was 1.078 (95\% confidence interval 0.950 to $1.224 ; \mathrm{P}=0.243)$. With respect to meat and meat products and dairy products, as expected we found positive associations, which for meat and meat products approached statistical significance $(\mathrm{P}=0.06)$. Because ethanol was introduced in the Mediterranean diet score as a binary variable (moderate intake versus other), we also calculated the corresponding mortality ratio, which is 0.810 (0.706 to 0.931$)$.

After a mean follow-up of 8.5 years, 652 deaths from any cause had occurred among 12694 participants with Mediterranean diet scores 0-4 and 423 among 10655 participants with scores 5 or more. Table 4 shows the mortality ratio associated with a two unit increment in the Mediterranean diet score, as well as how this ratio changes with alternate exclusion of each of the nine components of the score. Because of the construction of the score (in which one unit is assigned when a beneficial component is consumed in high quantities, ethanol is consumed in moderate quantities, or a non-beneficial component is consumed in low quantities), the benefit is expected to decrease (and the mortality ratio is expected to increase towards the null value of 1) after the alternate exclusion from the score of each of the nine components. Table 4 also shows the percentage reduction in the apparent effect of the Mediterranean diet score. Thus, when vegetables are excluded from the score the mortality ratio, properly adjusted for the change from a 10 point to a nine point scale, increases from 0.864 to 0.886 - that is, the beneficial effect of the Mediterranean diet score is reduced from $1-0.864=0.136$ to $1-0.886=0.114$ or by $16.2 \%$. In the construction of the Mediterranean diet 
score, fish and seafood are expected to have an inverse (beneficial) association, but in this particular dataset the association turned out to be non-significantly positive $\left(\right.$ table $3^{3}$ ). For this reason, we did not calculate the mortality ratio corresponding to the exclusion of fish and seafood from the Mediterranean diet score in table 4 .

Table 5 shows changes in the mortality ratios associated with a two unit increment in the Mediterranean diet score (properly adjusted for the sequential change in scale), after successive removal of each of its components, ranked according to the magnitude of effect in the model in which the nine components were mutually adjusted (table 3). Again, we did not consider fish and seafood in this analysis (except as a possible confounding variable). As expected, the mortality ratio gradually approaches the null value of 1 after removal firstly of ethanol, then of meat and meat products, then of vegetables, then of fruits and nuts, then of the lipid ratio, and finally of legumes. Change in the order of removal did not noticeably affect the pattern shown in table 5 .

We also examined the consequences of the joint presence of any two by two combinations of the nine components of the Mediterranean diet score, excluding combinations of fish and seafood with the rest of the components because of the unexpected (probably owing to chance) positive association of this food group with mortality. Of the 28 possible two by two combinations, we found clear additive or superadditive associations of the joint presence of any two components in 13 instances (in none of these instances was there a statistically significant interaction in the multiplicative scale implicit in the Cox regression). Moderate ethanol consumption, high lipid ratio, and low intake of meat and meat products were each present five times in the $26(2 \times 13)$ possible entries, whereas high intakes of vegetable, fruits, and legumes were each represented three times (the remaining two entries were once for low intake of dairy products and once for high cereal intake). In conjunction with the results in table 5 , we interpret these findings as suggesting that moderate ethanol intake, low intake of meat and meat products, high lipid ratio, and high intake of plant foods are driving the association of high Mediterranean diet score with low mortality.

\section{DISCUSSION}

\section{Main findings}

In a prospective investigation based on the general population, in which 23349 participants were followed up for 199726 person years and 1075 deaths occurred, we found that people with closer adherence to the traditional Mediterranean diet, as indicated by the Mediterranean diet score, had lower overall mortality. Specifically, increased adherence to the Mediterranean diet score by two units, a realistic change, was associated with a statistically significant $14 \%$ lower overall mortality. In many studies assessing the health effects of the Mediterranean diet, the authors have speculated about the biological processes that mediate its apparent effects, focusing on possible antioncogenic actions of the oleic acid in olive oil, ${ }^{18}$ resveratrol and piceid mostly in wine, ${ }^{19}$ and several other antioxidants in olive oil and plant foods, ${ }^{20}$ as well as the widely accepted favourable effects of olive oil on blood lipids. ${ }^{21}$

The contribution of the nine components to the Mediterranean diet score was approximately additive in the sense that the addition of the excesses or the deficits in the mortality ratios in table 3 divided by the number of components (nine) would equal -0.07 and would approximately correspond to the mortality ratio of the Mediterranean diet score per one unit increment. Nevertheless, the contribution to the association of adherence to the Mediterranean diet score with lower mortality was larger for moderate (rather than excessive or minimal) consumption of ethanol (24\%), followed by low consumption of meat and meat products $(17 \%)$ and high consumption of vegetables $(16 \%)$; high consumption of fruits and nuts, high monounsaturated to saturated lipid ratio, and high consumption of legumes each contributed about $10-11 \%$. The contributions of high consumption of cereals and low

Table $4 \mid$ Mortality ratios associated with two unit increment* in Mediterranean diet score (MDS) and after alternate subtraction of each of its dietary components

\begin{tabular}{|c|c|c|c|}
\hline Dietary variable & Mortality ratio $(95 \% \mathrm{Cl})$ & $P$ value & Reduction in apparent effect (\%)‡ \\
\hline MDS overall & 0.864 (0.802 to 0.932$)$ & $<0.001$ & 0 \\
\hline MDS minus vegetables & $0.886(0.822$ to 0.955$)$ & 0.002 & 16.2 \\
\hline MDS minus legumes & 0.877 (0.815 to 0.944$)$ & $<0.001$ & 9.7 \\
\hline MDS minus fruit and nuts & 0.879 (0.818 to 0.946$)$ & 0.001 & 11.2 \\
\hline MDS minus cereals & $0.872(0.814$ to 0.935$)$ & $<0.001$ & 6.1 \\
\hline MDS minus monounsaturated/saturated lipids (ratio) & $0.878(0.806$ to 0.957$)$ & 0.003 & 10.6 \\
\hline MDS minus dairy products & 0.870 (0.806 to 0.939$)$ & $<0.001$ & 4.5 \\
\hline MDS minus meat and meat products & 0.887 (0.825 to 0.953$)$ & 0.001 & 16.6 \\
\hline MDS minus ethanol & $0.896(0.835$ to 0.962$)$ & 0.002 & 23.5 \\
\hline
\end{tabular}

${ }^{*}$ Originally estimated logarithms of mortality ratios were multiplied by $9 / 10$ and then exponentiated to correct for nine point scale.

$\dagger$ Adjusted for sex, age $(445,45-54,55-64, \geq 65$, categorically), education (none/elementary school degree, secondary or technical school degree, university degree or higher, categorically), smoking status (never, former, and current at enrolment with cigarettes per day 1-10, 11-20, 21-30, 31-40, $\geq 41$ ordered), waist:hip ratio (sex specific fifths, ordered), body mass index (sex specific fifths, ordered), MET score (fifths, ordered), total energy intake (fifths, ordered), and corresponding subtracted components («median intake, $\geq$ median intake). $\ddagger$ Estimated from original numbers. 
Table 5| Mortality ratios associated with two unit increment in Mediterranean diet score after successive removal of each of its components

\begin{tabular}{lll} 
Dietary variable & Mortality ratio* $(95 \% \mathrm{Cl})$ & P value \\
Mediterranean diet score overall & $0.864(0.802$ to 0.932$)$ & 0.001 \\
\hline Minus ethanol & $0.896 \dagger(0.835$ to 0.962$)$ & 0.002 \\
\hline And minus meat and meat products & $0.919 \ddagger(0.859$ to 0.983$)$ & 0.014 \\
\hline And minus vegetables & $0.945 \S(0.884$ to 1.009$)$ & 0.091 \\
\hline And minus fruit and nuts & $0.959 \uparrow(0.903$ to 1.020$)$ & 0.181 \\
\hline And minus monounsaturated/saturated & $0.986^{\star \star}(0.922$ to 1.054$)$ & 0.678 \\
\hline lipids (ratio) & & 0.985 \\
\hline And minus legumes & $1.001 \dagger \dagger(0.942$ to 1.063$)$ & \\
\hline
\end{tabular}

*Adjusted for sex, age ( $<45,45-54,55-64, \geq 65$, categorically), education (none/elementary school degree,

secondary or technical school degree, university degree or higher, categorically), smoking status (never, former, and current at enrolment with cigarettes per day 1-10, 11-20, 21-30, 31-40, $\geq 41$ ordered), waist:hip ratio (sex specific fifths, ordered), body mass index (sex specific fifths, ordered), MET score (fifths, ordered), total energy intake (fifths, ordered), and all dietary components that have been removed («median intake, zmedian intake).

†Originally estimated logarithm of mortality ratio was multiplied by $9 / 10$ and then exponentiated to adjust to

10 point scale.

$\ddagger$ Originally estimated logarithm of mortality ratio was multiplied by $8 / 10$ and then exponentiated to adjust to 10 point scale.

§Originally estimated logarithm of mortality ratio was multiplied by $7 / 10$ and then exponentiated to adjust to 10 point scale.

TOriginally estimated logarithm of mortality ratio was multiplied by $6 / 10$ and then exponentiated to adjust to 10 point scale.

** Originally estimated logarithm of mortality ratio was multiplied by $5 / 10$ and then exponentiated to adjust to 10 point scale.

††Originally estimated logarithm of mortality ratio was multiplied by $4 / 10$ and then exponentiated to adjust to 10 point scale. meat and meat products, and the lipid ratio; to a lesser extend to vegetables, fruits, and legumes; and only exceptionally to cereals and dairy products.

\section{Advantages of Mediterranean diet score}

Certain questions need to be considered in view of these results. Why do Mediterranean diet scores tend to generate fairly consistent results with respect to health benefits, whereas studies focusing on the component foods or food groups are often contradictory? Why do Mediterranean diet scores in Mediterranean countries seem to be more strongly inversely related to adverse health outcomes than Mediterranean-like scores in non-Mediterranean countries? With respect to the first question, chance, non-differential misclassification, and residual confounding may have more important consequences when a single food is evaluated rather than a multi-component, unidimensional score, for several reasons. ${ }^{723}$ Thus, chance is more likely to disruptively operate on a single food group rather than simultaneously on several components on a score. Moreover, in analysis focusing on individual components, effects are examined against the background of average risk associated with other nutritional components, whereas a dietary score can account for extremes of cumulative exposures in the absence of other major nutritional effects. With respect to the second question, the Mediterranean diet scores in Mediterranean countries assess the impact of large quantities of fruits and nuts, vegetables, legumes, and olive oil, which are consumed by many people in Mediterranean countries (in fact, the high consumption of olive oil facilitates the high consumption of vegetables and legumes) but by relatively few people in non-Mediterranean countries (the medians used as cut-offs are generally study specific). Also, in Mediterranean countries ethanol is mostly consumed in the form of wine and during meals, and reports now suggest that even white wines contain antioxidant compounds and that wine may modulate the health effects of other nutritional compounds in the stomach. $^{24-27}$ is probably better than that of habitual intake of the foods contributing to the eight other components, and the corresponding non-differential misclassification (and thus attenuation of a true association) is likely to be less evident for alcohol than for the other components. Moreover, solid evidence exists for a U-shaped association of alcohol with total mortality, ${ }^{22}$ dictating the use of two cut-off points and, thus, allowing extraction of more informative results about the health effects of this component. Cereals, as generally reported, are a mix of the wholegrain cereals (which are presumed to be beneficial) and other varieties, and milk and dairy products are also a mix of low fat and full fat products (which are presumed to have differential effects on health). Lastly, consumption of fish and seafood by the participants in this study was generally quite low. Nevertheless, super-additive or clearly additive effects with respect to mortality of two by two combinations of the nine components of the Mediterranean diet score refer mostly to ethanol,

\section{Strengths and limitations of study}

Advantages of this study are its prospective nature, its reliance on a population based sample in a typical Mediterranean country, and the use of a validated dietary questionnaire administered by an interviewer. A limitation is that diet was assessed many years before the occurrence of the outcome, allowing for unavoidable intervening changes in dietary habits. This is a likely reason for the apparently weaker association between Mediterranean diet score and mortality in these data in comparison with an earlier analysis in the same cohort, ${ }^{7}$ although other methodological reasons could also be invoked. ${ }^{28}$ Power constraints did not allow us to look for multiple disease specific associations, but, in any case, assessing what is a beneficial diet overall rather than a diet focusing on a specific disease (or group of diseases) is an obvious priority. We were unable to evaluate in a meaningful way 


\section{WHAT IS ALREADY KNOWN ON THIS TOPIC}

Several prospective cohort studies have consistently indicated that conformity to the traditional Mediterranean diet is associated with longevity

No study has investigated the relative importance of individual components of the Mediterranean diet score in the generation of this association

\section{WHAT THIS STUDY ADDS}

The contribution of the nine components to the apparent protective effect of the score assessing adherence to traditional Mediterranean diet is approximately additive

The dominant components of the Mediterranean diet score as a predictor of lower mortality are moderate consumption of alcohol, low consumption of meat and meat products, and high consumption of vegetables, fruits and nuts, olive oil, and legumes

multiplicative interactions, again because of power constraints. Moreover, when the mortality ratio varies by at most around 1.5, the excess risk from an additive or multiplicative joint action of two factors is fairly similar (additive effect 2.0, multiplicative effect 2.25). Thus, the results of our study do not refute the possibility of synergistic effects among foods and nutrients in the Mediterranean diet.

\section{Conclusions}

An analysis of this type cannot provide universally applicable results, because diet varies across populations and also between sections of the same population. Nevertheless, our results indicate that the Mediterranean diet score that has been widely used is an effective predictor of mortality because it integrates associations with mortality of many individual components in a single unidimensional score. The analysis also indicates that the dominant components of the Mediterranean diet score as a predictor of lower mortality are moderate consumption of ethanol (mostly in the form of wine during meals, as is traditional in the Mediterranean countries), low consumption of meat and meat products, and high consumption of vegetables, fruits and nuts, olive oil, and legumes. In contrast, high consumption of fish and seafood and cereals overall, as well as low consumption of dairy products overall, as components of the traditional Mediterranean diet contribute little to the ability of the Mediterranean diet score to predict mortality, at least in this Mediterranean population.

Contributors: AT is the principal investigator of the Greek EPIC study. She was responsible for the conception and design of the study, data collection, interpretation of results, and drafting the manuscript. CB was responsible for the statistical analysis and interpretation of results and drafting the manuscript. DT was the senior epidemiologist and contributed to study design and analysis and the drafting of the manuscript. All authors have seen and approved the final version of the manuscript. AT is the guarantor.

Funding: This study was supported by the Europe against Cancer Program of the European Commission, the Greek Ministries of Health and Education, and a grant to the Hellenic Health Foundation by the Stavros
Niarchos Foundation. The study sponsors did not have any role in the study design; the collection, analysis, and interpretation of data; the writing of the report; or the decision to submit the paper for publication. Researchers were independent from funders.

Competing interests: None declared.

Ethical approval: All volunteers signed informed consent forms, and the study protocol was approved by ethics committees at the International Agency for Research on Cancer (Lyon, France) and the University of Athens, Medical School.

1 Keys A. Seven countries: a multivariate analysis of death and coronary heart disease. Cambridge, MA: Harvard University Press, 1980.

2 Trichopoulou A, Kouris-Blazos A, Wahlqvist ML, Gnardellis C, Lagiou P, Polychronopoulos E, et al. Diet and overall survival in the elderly. BMJ 1995;311:1457-60.

3 Scarmeas N, Stern Y, Mayeux R, Luchsinger JA. Mediterranean diet, Alzheimer disease, and vascular mediation. Arch Neurol 2006:63:1709-17.

4 Martínez-González MA, de la Fuente-Arrillaga C, Nunez-Cordoba JM, Basterra-Gortari FJ, Beunza JJ, Vazquez Z, et al. Adherence to Mediterranean diet and risk of developing diabetes: prospective cohort study. BMJ 2008;336:1348-51.

5 Benetou V, Trichopoulou A, Orfanos P, Naska A, Lagiou P, Boffetta P, et al. Conformity to traditional Mediterranean diet and cancer incidence: the Greek EPIC cohort. Br I Cancer 2008;99:191-5.

6 Sofi F, Cesari F, Abbate R, Gensini GF, Casini A. Adherence to Mediterranean diet and health status: meta-analysis. $B M$ J 2008;337:a1344.

7 Trichopoulou A, Costacou T, Bamia C, Trichopoulos D. Adherence to a Mediterranean diet and survival in a Greek population. NEngl JMed 2003;348:2599-608.

8 Lasheras C, Fernandez S, Patterson AM. Mediterranean diet and age with respect to overall survival in institutionalized, nonsmoking elderly people. Am J Clin Nutr 2000;71:987-92.

9 Trichopoulou A, Orfanos P, Norat T, Bueno-de-Mesquita B, Ocké MC, Peeters PH, et al. Modified Mediterranean diet and survival: EPIC elderly prospective cohort study. BMJ 2005;330:991.

10 Lagiou P, Trichopoulos D, Sandin S, Lagiou A, Mucci L, Wolk A, et al. Mediterranean dietary pattern and mortality among young women: a cohort study in Sweden. Br J Nutr 2006;96:384-92.

11 Riboli E, Hunt KJ, Slimani N, Ferrari P, Norat T, Fahey M, et al. European Prospective Investigation into Cancer and Nutrition (EPIC): study populations and data collection. Public Health Nutr 2002;5:1113-24.

12 Slimani N, Kaaks R, Ferrari P, Casagrande C, Clavel-Chapelon F, Lotze G, et al. European Prospective Investigation into Cancer and Nutrition (EPIC) calibration study: rationale, design and population characteristics. Public Health Nutr 2002;5:1125-45.

13 Katsouyianni K, Rimm EB, Gnardellis C, Trichopoulos D, Polychronopoulos E, Trichopoulou A. Reproducibility and relative validity of an extensive semi-quantitative food frequency questionnaire using dietary records and chemical markers among Greek school teachers. Int I Epidemiol 1997;26(suppl 1):S118-27.

14 Trichopoulou A, Georga K. Composition tables of simple and composite foods. Athens: Parisianos, 2002.

15 Trichopoulou A, Gnardellis C, Lagiou P, Benetou V, Trichopoulos D. Body mass index in relation to energy intake and expenditure among adults in Greece. Epidemiology 2000;11:333-6

16 Bamia C, Trichopoulou A, Lenas D, Trichopoulos D. Tobacco smoking in relation to body fat mass and distribution in a general population sample. Int J Obes Relat Metab Disord 2004;28:1091-6.

17 Benetou V, Bamia C, Trichopoulos D, Mountokalakis T, Psaltopoulou T, Trichopoulou A. The association of body mass index and waist circumference with blood pressure depends on age and gender: a study of 10,928 non-smoking adults in the Greek EPIC cohort. Eur J Epidemiol 2004;19:803-9.

18 Menendez JA, Lupu R. Mediterranean dietary traditions for the molecular treatment of human cancer: anti-oncogenic actions of the main olive oil's monounsaturated fatty acid oleic acid (18:1n-9). Curr Pharm Biotechnol 2006; 7:495-502.

19 Zamora-Ros R, Andres-Lacueva C, Lamuela-Raventós RM, Berenguer T, Jakszyn P, Martínez C, et al. Concentrations of resveratrol and derivatives in foods and estimation of dietary intake in a Spanish population: European Prospective Investigation into Cancer and Nutrition (EPIC)-Spain cohort. Br J Nutr 2008;100:188-96

20 Dedoussis GV, Kanoni S, Mariani E, Cattini L, Herbein G, Fulop T, et al. Mediterranean diet and plasma concentration of inflammatory markers in old and very old subjects in the ZINCAGE population study. Clin Chem Lab Med 2008;46:990-6.

21 Mensink RP, Katan MB. Effect of monounsaturated fatty acids versus complex carbohydrates on high-density lipoproteins in healthy men and women. Lancet 1987;1:122-5. 
22 Di Castelnuovo A, Costanzo S, Bagnardi V, Donati MB, lacoviello L, de Gaetano G. Alcohol dosing and total mortality in men and women. Arch Intern Med 2006;166:2437-45.

23 Jacques PF, Tucker KL. Are dietary patterns useful for understanding the role of diet in chronic disease? Am J Clin Nutr 2001;73:1-2.

24 Pignatelli P, Ghiselli A, Buchetti B, Carnevale R, Natella F, Germanò G, et al. Polyphenols synergistically inhibit oxidative stress in subjects given red and white wine. Atherosclerosis 2006;188:77-83.

25 RajdI D, Racek J, Trefil L, Siala K. Effect of white wine consumption on oxidative stress markers and homocysteine levels. Physiol Res 2007;56:203-12.
26 Gorelik S, Ligumsky M, Kohen R, Kanner J. The stomach as a "bioreactor": when red meat meets red wine. J Agric Food Chem 2008;56:5002-7.

27 Kanner J, Lapidot T. The stomach as a bioreactor: dietary lipid peroxidation in the gastric fluid and the effects of plant-derived antioxidants. Free Radic Biol Med 2001;31:1388-95.

28 loannidis JP. Why most discovered true associations are inflated. Epidemiology 2008;19:640-8.

Accepted: 6 February 2009 